\title{
IR783 Encapsulated in TR-conjugated Liposomes for Enhancing NIR Imaging-guided Photothermal and Photodynamic Therapy
}

Jiajia Lv

Zunyi Medical University

Tianjiao Luan

Zunyi Medical University

Mingyan Yang

Zunyi Medical University

Mengmeng Wang

Zunyi Medical University

Jie Gao

Zunyi Medical University

Zeli Yuan ( $\nabla$ zlyuan@zmu.edu.cn )

Zunyi Medical University https://orcid.org/0000-0001-5354-769X

\section{Research Article}

Keywords: liposome, RGD peptide, photothermal therapy, photodynamic therapy, NIR imaging guided

Posted Date: November 9th, 2021

DOI: https://doi.org/10.21203/rs.3.rs-1033993/v1

License: (9) (i) This work is licensed under a Creative Commons Attribution 4.0 International License.

Read Full License 
$1 \quad$ IR783 encapsulated in TR-conjugated liposomes for

2 enhancing NIR imaging-guided photothermal and

3 photodynamic therapy

4 Jiajia Lv ${ }^{1,2,3,4}$, Tianjiao Luan ${ }^{1,2,3,4}$, Mingyan Yang ${ }^{1,2,3,4}$, Mengmeng Wang ${ }^{1,2,3,4}$, Jie Gao ${ }^{1,2,3,4 *}$, Zeli

5 Yuan $^{1,2,3,4 *}$

6 Abstract

7 We developed an integrin avß3-specific liposomes, TR-conjugated liposomes (TR-LPs), loading

8 IR783 for NIR imaging-guided both PTT and PDT. The TR-LPs was composed of

9 soyabeanphosphatidylcholine, cholesterol, 1,2-distearoyl-sn-glycero-3-phosphoethanolamine- N-

10 [methoxy(polyethylene glycol)-2000] (DSPE-PEG) and TR-functionalized DSPE-PEG. IR783, NIR

11 PTT/PDT diagnostic agents, were encapsulated in the hydrophilic core of the TR-LPs. DSPE-

12 PEG had ability of reducing the absorption of TR-LPs by the reticuloendothelial system and

13 increase the cycle time in body. RGD fragment on the TR peptide (TR $=c(R G D)$ -

14 AGYLLGHINLHHLAHL(Aib)HHIL-cys) enhanced the tumor selectivity of liposomes by specifically

15 targeting integrin $\alpha v \beta 3$-overexpressing cancer cells. Simultaneously, the rest of fragment on the

16 TR peptide can be changed to the positive charge in the tumor microenvironment $(\mathrm{pH} 6.5)$,

17 improving cellular uptake of photoagents at tumor site. We executed a set of in vitro and in vivo

18 experiments to verify if, by functionalizing liposomes with an integrin $\alpha \mathrm{v} \beta 3$-specific and $\mathrm{pH}$

19 responding peptide, it is possible to achieve NIR imaging guided PTT/PDT for tumor treatment.

20 TR-conjugated liposomes exhibited favorable physical and chemical stability, loading capacity,

21 biocompatibility and tumor targeting. TR-LPs can safely and efficiently delivery IR783 to tumor

22 sites to achieve their therapeutic function. IR783-TR-LPs is promising as a potentially safe and

23 effective phototherapeutic agents for NIR fluorescence-guided tumor therapy applications.

24 Keywords: liposome, RGD peptide, photothermal therapy, photodynamic therapy, NIR imaging-

25 guided 


\section{Background}

28 Currently, phototherapy advancements have gradually shifted from monotherapy to multimodal

29 therapy, typically combining photodynamic therapy (PDT) and photothermal therapy (PTT) into

30 one system [1-6]. PDT mainly relies on photosensitizers (PS) under light irradiation to directly

31 generate reactive oxygen species (ROS) to kill cells, so suitable irradiation wavelength and

32 effective PS delivery is crucial to achieving excellent PDT efficacy [7]. PTT can not only generate

33 hyperthermia to kill cancer cells, but also increase the saturated oxygen concentration and the

34 amount of drug delivered by accelerating blood flow [8,9]. Typically, hyperthermia is able to

35 enhance the absorption of PS by promoting the permeability of cell membrane, and finally

36 increase the concentration of PS in cancer cells [6]. More importantly, phototherapy can achieve

37 fluorescence navigation to accurately activate PTT and PDT at tumor tissue and avoid normal

38 tissue damage. Therefore, the combination of fluorescence imaging-guided PTT and PDT is a

39 promising non-invasive method for cancer treatment.

40 The common method for developing multimodal phototherapy is mainly loading two or more

41 PDT and PTT agents in one nanosystem. The strategy may face the following issues: (1) different

42 lasers to activate treatment resulting complicated operation, device/time cost, synergistic

43 therapeutic destruction, (2) complex preparation, and (3) challenges in obtaining optimal ratio of

44 PTT and PDT agents to achieving synergistic effects. If both PDT and PPT agents can be

45 activated simultaneously by the same light irradiation, it will unify the treatment time of PDT and

46 PTT, reduce the complexity of treatment, as well as be more conducive to achieving optimal

47 synergy. Thus, it is critical to develop a mono-phototherapy agent combing PTT and PDT that can

48 simultaneously perform tumor-specific targeting, real-time fluorescence tracking in vivo and

49 selective tumor ablation.

$50 \quad$ Near-infrared (NIR) irradiation and fluorescence imaging as emerging treatment technologies

51 have used in fundamental research and clinical application, for the advances in increasing issue

52 penetration depth, reducing tissue absorption, photon scattering, and tissue autofluorescence

53 interference [10]. Owing to advances in excellent biodegradability, biocompatibility and low 
54 toxicity, NIR organic dyes have received extensive attention. Indocyanine green (ICG), as an important derivative of cyanine dye, has been approved by the FDA for medical imaging in clinical

56 practice. Therefore, many NIR fluorescent dyes studied are based on cyanine dye family. In

57 addition to NIR fluorescence imaging, ICG is proven to have PTT and PDT capabilities [11].

58 However, since ICG is unstable in an aqueous medium and the fluorescence quantum yield is

59 relatively low, which significantly reduces its imaging and therapy potential. Thus, various cyanine

60 dyes were developed to improve the stability and photophysical properties of ICG. One of these

61 strategies is the introduction of a rigid group in the middle of cyanine dyes, which can greatly

62 improve the photophysical properties of cyanine dyes [12]. Typically, IR783 with a rigid

63 carbocyclic ring exhibits that preferable aqueous stability and fluorescence quantum yield in

64 comparison to ICG. IR783, a water-soluble cyanine dye, often demonstrates similar

65 biodistribution profile to ICG after systemic administration and rapid clearance from systemic

66 circulation [13]. IR783 is characterized by a short tumor retention time due to its rapid clearance,

67 which greatly limits the time window in which phototherapy can be administered.

68 Liposomes, as the most successful nanocarrier in clinical applications, have been applied to

69 a variety of commercial nanomedicines including Doxil, Caelyx and so all [14]. Although

70 traditional liposomes have an enhanced permeability and retention (EPR) effect, the amount of

71 enrichment at the tumor site is still limited. Recently, RGD (Arg-Gly-Asp) peptide functionalized

72 liposomes is use to improve their tumor targeting due to the excellent specificity of RGD for av $\beta 3$

73 integrin positive cells and tumor angiogenic vessels $[15,16]$. He and co-workers developed

74 integrin $\alpha \mathrm{v} \beta 3$-specific liposomes which respond to $\mathrm{pH}$ and cross the blood-brain barrier to treat

75 glioma [17]. Gu and co-workers used quantum dots and RGD-functionalized polyethylene glycol

76 (PEG) to construct sub-50 nm nanoparticles for inhibition of both primary and metastatic cancer

77 [18]. Lin and co-workers designed a multimodal diagnostic nanoplatform for pohotothermal-

78 enhanced chemotherapy of tumor, which was composed RGD peptide, melanin-coated magnetic

79 nanoparticles, doxorubicin, and ICG [19].

80 In this work, we developed an integrin $\alpha v \beta 3$-specific liposomes, TR-conjugated liposomes

81 (TR-LPs), loading IR783 for NIR imaging-guided both PTT and PDT. The TR-LPs was composed 
of soyabeanphosphatidylcholine (SPC), cholesterol (Cho), 1,2-distearoyl-sn-glycero-3-

83 phosphoethanolamine-N-[methoxy(polyethylene glycol)-2000] (DSPE-PEG) and TR-

84 functionalized DSPE-PEG. IR783, NIR PTT/PDT diagnostic agents, were encapsulated in the

85 hydrophilic core of the TR-LPs. DSPE-PEG can reduce the absorption of TR-LPs by the reticuloendothelial system and increase the cycle time in body. RGD fragment on the TR peptide

$87(T R=c(R G D)-A G Y L L G H I N L H H L A H L(A i b) H H I L-c y s)$ enhanced the tumor selectivity of liposomes by specifically targeting integrin avß3-overexpressing cancer cells. Simultaneously, the rest of fragment on the TR peptide can be changed to the positive charge in the tumor microenvironment (pH 6.5), improving cellular uptake of photoagents at tumor site. We executed a set of in vivo and in vivo experiments to verify if, by functionalizing liposomes with an integrin $\alpha v \beta 3$-specific and $\mathrm{pH}$ responding peptide, it is possible to achieve NIR imaging guided PTT/PDT for tumor treatment.

\section{Results and discussion}

\section{Preparation and characterization of IR783-TR-LPs}

95 IR783-TR-LPs were composed of SPC, Cho, DSPE-PEG and TR-DSPE-PEG. The IR783 were encapsulated in the hydrophilic core of the liposome nanoparticles (Scheme 1). DSPE-PEG had ability of reducing the absorption of IR783-TR-LPs by the reticuloendothelial system and increase the cycle time in body. Moreover, RGD fragment on TR peptide enhanced the specific targeting of liposomes to cancer cells overexpressing integrin $\alpha v \beta 3$. Simultaneously, the rest of fragment on the TR peptide can be changed to the positive charge in the tumor microenvironment, improving cellular uptake of liposomes at tumor site [17].

As presented in Fig. 1 and Table 1, the hydrodynamic size of the TR-LPs was measured to be $104.4 \mathrm{~nm}$ at $\mathrm{pH} 6.5$ and $143.3 \mathrm{~nm}$ at $\mathrm{pH}$ 7.4. The reduction in particle size of the TR-LPs at $\mathrm{pH}$ 6.5 will facilitate tissue penetration [20]. Compared to TR-LPs, PEG-LPs had no change in the hydrodynamic size at pH 6.5 and 7.4. In addition, encapsulation efficiency of TR-LPs was

107 acting as a nanovehicle for loading IR783. Furthermore, the zeta potential of TR-LPs and PEG-

108 LPs was analyzed by a Zeta PALS instrument. The PEG-TPs possessed a negative surface 
potential of $-18.15 \mathrm{mV}$ at $\mathrm{pH} 6.5$ and $-16.00 \mathrm{mV}$ at pH 7.4. After assembling with TR-DSPEPEG, the zeta potential rose to $-7.83 \mathrm{mV}$ at $\mathrm{pH}$ 7.4. At $\mathrm{pH}$ 6.5, TR-DSPE-PEG significantly enhanced the surface potential of TR-LPs, which was measured to be $+5.09 \mathrm{mV}$. The change of zeta potential showed that TR-LPs could undergo charge reversal (the zeta potential changing from negative to positive) under slightly acidic conditions, which was beneficial to cellular uptake 114 in tumor microenvironment.

115 For subsequent biological applications, we investigated the stability of TR-LPs and PEG-LPs 116 in PBS (pH 7.4), DMEM and 10\% FBS (FBS:PBS, v:v) by DLS (Fig. 1C and Fig. S1). The particle 117 size of TR-LPs fell in the range of about $110 \mathrm{~nm}$ without significant change in three different 118 media for 24 hours. Similarly, the particle size of PEG-TPs was relatively stable in these three 119 solutions for 24 hours. Finally, TR-LPs and PEG-LPs presented stability in PBS, DMEM and 10\% 120 FBS with no precipitation stored in dark, and $4{ }^{\circ} \mathrm{C}$ for storing at least one week.

121 As shown in Fig. 1D, UV-Vis spectra showed that free IR783 possessed a strong absorption 122 peak at $\sim 776 \mathrm{~nm}$ in PBS. After IR783 encapsulated, the absorption spectrums of IR783-TR-LPs 123 and IR783-PEG-LPs had almost no change compared with free IR783. If the IR783 located in a 124 hydrophobic area of liposomes, its spectral absorption may change significantly due to changes 125 in solvent polarity. Furthermore, the absorbance spectrums of TR-LPs and PEG-LPs possessed 126 the characteristic peaks of IR783 with minor changes, illustrating that IR783 had been 127 successfully encapsulated and located at hydrophilic core of the liposome nanoparticles.

128 Free IR783, IR783-PEG-LPs and IR783-TR-LPs were separately stored under irradiation of 129 LED light (white light, $6.78 \mathrm{~W}$ ) at room temperature to investigate the photostability of them. The 130 UV-Vis spectra of free IR783, IR783-PEG-LPs and IR783-TR-LPs were recorded at a specific 131 time point for 47 min. Finally, the half-life $\left(\mathrm{t}_{1 / 2}\right)$ of free IR783, IR783-PEG-LPs and IR783-TR-LPs 132 were $12.6 \mathrm{~min}, 14.2 \mathrm{~min}$ and $16.4 \mathrm{~min}$ by fitting the maximum absorbance IR783 (Fig. 1E). 133 Obviously, IR783-TR-LPs showed better stability than IR783-PEG-LPs and free IR783. Thus, the 134 relative photostability of IR783-TR-LPs was helpful for subsequent experiments in vitro and in 135 vivo. 
In the release experiment of IR783, IR783-TR-LPs exhibited more cumulative release of IR783 at pH $6.5(68.2 \%)$ than that of pH $7.4(55.0 \%)$ after $24 \mathrm{~h}$ (Fig. 1F and Fig. S2). IR783, being both a photothermal and a photodynamic agent, its ability to be released from liposomes did not affect its ability to exert phototherapeutic effects in our system. Ultimately, That the ability of IR783 loading with above liposomes to achieve maximum enrichment at the tumor site is key to achieving optimal PTT/PDT effects.

\section{Phototheranostic properties}

143 To achieve optimal imaging and therapeutic results in vivo, it is critical that IR783 has good 144 photophysical properties in NIR region. IR783, as cyanine dye-based phototheranostic agents, 145 dissolved in PBS buffer exhibit NIR maximum absorption $(\sim 776 \mathrm{~nm})$ and fluorescence emission 146 ( 794 nm). Fluorescence quantum yields $\left(\Phi_{\mathrm{F}}\right)$ for IR783-TR-LPs and IR783-PEG-LPs were calculated in PBS buffer using IR783 as the reference ( $\Phi_{\mathrm{F}}$ was $18.6 \%$ in PBS buffer, pH 7.4) [21].

148 Fluorescence quantum yields of the formulated IR783-TR-LPs and IR783-PEG-LPs were 17.2\% 149 and $16.9 \%$, while maintaining phototherapeutic abilities. Noteworthy, $\Phi_{F}$ of IR783-TR-LPs and 150 IR783-TR-LPs was reduced to $7.5 \%$ and $9.1 \%$, presumably due to aggregation of IR783 in the 151 hydrophilic core of the liposomes resulting fluorescence quenching effect.

152 To assess photothermal properties of the free IR783, IR783-TR-LPs and IR783-PEG-LPs in 153 PBS buffer at different IR783 concentration $(0,0.25,0.5,1 \mathrm{mM})$ exposed to NIR laser light (808 $\left.154 \mathrm{~nm}, 2 \mathrm{~W} / \mathrm{cm}^{2}\right)$ or at the same IR783 concentration $(0.5 \mathrm{mM})$ exposed to laser irradiation $(0,1,2,3$ $155 \mathrm{~W} / \mathrm{cm}^{2}$ ) for $10 \mathrm{~min}$ and temperature profiles were obtained (Fig. 2A, 2B and Fig. S3). The results 156 revealed that IR783, the free IR783, IR783-TR-LPs and IR783-TR-LPs (at different IR783 157 concentration) all exhibited a rapid increase in temperature during exposure to NIR laser light $158\left(808 \mathrm{~nm}, 2 \mathrm{~W} / \mathrm{cm}^{2}\right)$, reaching about $50^{\circ} \mathrm{C}$ at $400 \mathrm{~s}$. Then, the temperature remained at $50^{\circ} \mathrm{C}$ for 159 the next $200 \mathrm{~s}$ and did not continue to rise. This was caused by the gradual photodegradation of 160 IR783, and it was also observed that the solution gradually changed from dark green to brown in 161 experiments. Finally, the photostability of IR783-TR-LPs, IR783-PEG-LPs and free IR783 was 162 also investigated. Toward this end, free IR783 and IR783 loaded by two liposomes were subject 163 to repeated irradiation-cooling cycles using $808 \mathrm{~nm}$ laser irradiation $\left(2 \mathrm{~W} / \mathrm{cm}^{2}\right)$ for $140 \mathrm{~min}$ 
followed by passive cooling to room temperature. As shown in Fig. 2C, 2D and Fig. S4, the variation in the maximal temperature increased during the whole cycle proved to be $1.9 \%$, leading us to conclude that only IR783-TR-LPs was stable under conditions of $808 \mathrm{~nm}$ laser irradiation. In contrast, the maximum temperature of PEG dropped significantly (from $74^{\circ} \mathrm{C}$ to $65.7^{\circ} \mathrm{C}$ ) in final irradiation cycle. After the fourth irradiation cycle of free IR783, the maximum temperature continued to drop $\left(73.6^{\circ} \mathrm{C} \rightarrow 53.2^{\circ} \mathrm{C} \rightarrow 25.8^{\circ} \mathrm{C}\right)$, and the temperature can no longer rise in the last cycle. These results proved that TR-LPs and PEG-LPs could protect IR783 from photobleaching, which was consistent with the previous experiment on stability of white light irradiation at room temperature.

In addition to the above optical and photothermal properties, the ROS generation of IR783 was also measured. After exposure to an $808 \mathrm{~nm}$ laser $\left(0.5 \mathrm{~W} / \mathrm{cm}^{2}\right)$ for $100 \mathrm{~s}$, IR783 capable of generating ROS, with respect to the dark controls and ROS scavenger (Fig. 2E).

Based on the above results, we selected IR783-TR-LPs, IR783-PEG-LPs and free IR783 for subsequent studies in vitro and in vivo. The above two liposomes (PEG-LPs and TR-LPs) both demonstrated enhanced photophysical and phototherapeutic properties compared to free IR783.

\section{In vitro phototherapy evaluation}

To demonstrate phototheranostic efficacy of IR783-TR-LPs, a 4T1 breast cancer cell line was used for in vitro studies. Upon exposure to $808 \mathrm{~nm}$ laser irradiation, Intracellular ROS generation of the IR783-TR-LPs, IR783-PEG-LPs and free IR783 can detect by DCFH-DA, which exhibit green fluorescence in 4T1 cells. As shown in Fig. 3A, the 4T1 cells treated by IR783-TR-LPs, IR783-PEG-LPs and free IR783 with NIR irradiation exhibit green fluorescence, demonstrating an amount of ROS generation in cells. Moreover, IR783-TR-LPs showed slightly higher fluorescence intensity and produces more ROS, which is consistent with the results of cell imaging. No fluorescence can be found in cells treated by the control groups of "no irradiation", "addition of $\mathrm{N}$ acetylcysteine with $808 \mathrm{~nm}$ laser irradiation", "cells treated with DCFH-DA under irradiation", "cells treated with DCFH-DA in dark", and "PBS".

Furthermore, in vitro PDT/PTT efficiency of IR783-TR-LPs, IR783-PEG-LPs and free IR783 in 4T1 cells was investigated by MTT assay. Two different power of laser $(800 \mathrm{~nm}, 0.1$ and 0.5 
$\left.\mathrm{W} / \mathrm{cm}^{2}\right)$ irradiating for 2 minutes were examined at the fixed concentration of IR783 (10 $\left.\mu \mathrm{M}\right)$ in different $\mathrm{pH}(\mathrm{pH} 7.4$ and $\mathrm{pH}$ 6.5) (Fig. 3B and 3C). Under the same $\mathrm{pH}$ conditions, the cell viability of IR783-TR-LPs was the lowest among the experimental groups after different power illumination. At pH 6.5, IR783-TR-LPs showed a better phototherapy effect. This is due to the encapsulation of free IR783, which enhanced the cell uptake and phototherapy of IR783. These results demonstrated that IR783-TR-LPs with NIR laser had ability of effectively killing tumor cells. According to the results of dark toxicity experiments (Fig. S5), the free IR783 was not toxic in the experimental concentration range, indicating good biocompatibility for subsequent cellular 200 and in vivo applications.

\section{In vivo imaging and phototheranostic study}

202 To evaluate the tumor targeting ability of IR783-TR-LPs, the in vivo biodistribution of IR783-TR203 LPs, IR783-PEG-LPs and free IR783 after tail vein injection were performed on BALB/c mice 204 bearing 4T1 tumor by a small animal NIR-imaging system. IR783-TR-LPs presented a much 205 stronger NIR-fluorescence signal in the tumor site at $8 \mathrm{~h}$ and $24 \mathrm{~h}$ compared with IR783-PEG206 LPs, which further demonstrated that TR ligand could effectively promote IR783 accumulating in 207 the tumor region in vivo (Fig. 4A). For IR783-TR-LPs, IR783 was predominantly cleared from the 208 tumor and body within 48 h. Compared with IR783-TR-LPs and IR783-PEG-LPs, free IR783 also 209 exhibited the property of preferential accumulation at the tumor area despite weaker fluorescence 210 intensity. Distinguished accumulation of free IR783 in the tumor was observed at $2 \mathrm{~h}$ until at $8 \mathrm{~h}$ 211 post injection, and IR783 was quickly cleared from the tumor and body within $24 \mathrm{~h}$. The results of

212 fluorescence imaging suggested that $8 \mathrm{~h}$ post injection was the optimal temporal window for 213 phototherapy.

214 Afterward, the major organs (heart, liver, spleen, lung and kidney) and tumor of BALB/c mice 215 were collected for ex vivo imaging at $8 \mathrm{~h}$. The fluorescence signal of IR783-TR-LPs group at the 216 tumor region was significantly higher than that of IR783-PEG-LPs and free IR783 group (Fig. 4A), 217 which was nearly quadruple and fifteen times using quantitative region-of-interest analysis,

218 respectively (Fig. S6). In addition, the fluorescence signal at the tumor site was approximately 219 double than that in liver or kidney after injection of IR783-TR-LPs after $8 \mathrm{~h}$. 
As presented in Fig. 4B, the tumor temperature of IR783-TR-LPs group at $8 \mathrm{~h}$ post injection elevated quickly from $30.3^{\circ} \mathrm{C}$ to $41.3^{\circ} \mathrm{C}$ with laser irradiation $\left(808 \mathrm{~nm}, 0.5 \mathrm{~W} / \mathrm{cm}^{2}\right)$ for $1 \mathrm{~min}$. However, IR783-PEG-LPs and free IR783 groups showed a relatively slight increase. Moreover, the tumor temperature of IR783-TR-LPs group increased to $42.0^{\circ} \mathrm{C}$ after laser irradiation for 5 min, which was higher than that of both IR783-PEG-LPs group $\left(41.1^{\circ} \mathrm{C}\right)$ and free IR783 $\left(39.5^{\circ} \mathrm{C}\right)$. As a control, the tumor temperature of PBS group was increased from $30.7^{\circ} \mathrm{C}$ to $37.7^{\circ} \mathrm{C}$ after laser irradiation for $5 \mathrm{~min}$. These results are also consistent with the results of NIR imaging, demonstrating the effectiveness of NIR imaging-guided phototherapy. Thus, IR783-TRLPs had the potential to be a promising NIR imaging-guided PDT/PTT agent for tumor treatment applications.

Encouraged by the high enrichment of IR783 from IR783-TR-LPs in tumor regions, we next studied both PDT and PTT efficiency in vivo. 4T1 tumor-bearing mice were tail-vein injected with 232 IR783-TR-LPs, IR783-PEG-LPs and free IR783 (1 mg/kg of IR783). In vivo study, 4T1 tumorbearing mice were randomly divided into four groups of five mice each, denoted as "IR783-TRLPs", "IR783-PEG-LPs", "free IR783" and "PBS". According to the results of NIR and photothermal imaging, the optimal therapeutic windows was determined at $8 \mathrm{~h}$ after injection for 5 min. Subsequently, All mice of four groups were exposed by $808 \mathrm{~nm}$ laser $\left(0.5 \mathrm{~W} / \mathrm{cm}^{2}\right)$ at $8 \mathrm{~h}$ after injection for $5 \mathrm{~min}$. The in vivo antitumor effects of the above photoagents were investigated by monitoring the changes in tumor volume for 15 days. As shown in Fig. 5A, the inhibition of tumor growth by treatment in the PBS group was negligible, indicating that $808 \mathrm{~nm}$ laser irradiation alone did not have an anti-tumor effect. It was revealed that treatment in "free IR783"

244 effective antitumor activity, as demonstrated by slow tumor growth at day 15 . Tumor photographs 245 further confirmed the optimal anti-tumor activity of the "IR783-TR-LPs" group, providing visual 246 evidence (Fig. 5B). In addition, negligible weight loss was observed in four groups of mice, 247 indicating low in vivo toxicity of the photo-treatments (Fig. 5C), which were further supported by 
the hematoxylin and eosin (H\&E) staining of liver and kidney tissues from all groups of each mouse, indicating the absence of significant lesions in normal organs (Fig. S7). On the 15th day, all four groups of mice were sacrificed to collect the tumor tissues, then sliced and stained with H\&E and TUNEL (in situ terminal deoxynucleotidyl transferased dUTP nick end labeling). The images histological sections demonstrate that among the different therapeutic agents, "IR783-TRLPs" group the most effective antitumor effect causing tumor necrosis, inducing apoptosis and hindering the proliferation of cancer cells (Fig. 5D). Additionally, the PEG-LPs and TR-LPs did not cause hemolysis in treatment (Fig. S8 and S9).

\section{Conclusion}

257 In summary (Figure 6), we have developed a versatile facile phototheranostic nanoagent based 258 on TR-conjugated liposomes loading IR783 for enhancing NIR imaging-guided PTT and PDT.

259 TR-conjugated liposomes exhibited favorable physical and chemical stability, loading capacity, 260 biocompatibility and tumor targeting. Guided by NIR fluorescence of IR783, we visualized the 261 tumor by NIR fluorescence in vivo to investigate the optimal time of IR783 enrichment and finally 262 achieved the optimal both PTT and PTT effect using NIR laser. As a comparison to both IR783263 PEG-LPs and free IR783, IR783-TR-LPs show the best treatment effect. These results clearly 264 indicate that TR-LPs, as a liposome, can safely and efficiently delivery IR783 to tumor sites to 265 achieve their therapeutic function. Meanwhile, with the help of TR-LPs, the physical and chemical 266 properties of IR783 were not only improved, but also its tumor selectivity was enhanced. IR783267 TR-LPs is promising as a potentially safe and effective phototherapeutic nanoagent for 268 fluorescence-guided tumor therapy applications. The above study is only as a proof of concept. In 269 the future, TR-LPs can be applied not only to load more other drugs than just photoagents to 270 achieve specific recognition of tumor cells, but even replace TR peptides with other targeting 271 molecules functionalized liposomes for achieving a wider range of medical applications.

\section{Material and methods}

\section{Materials}


274 Soyabeanphosphatidylcholine (SPC), cholesterol (Cho), IR783 (2-[2-[2-Chloro-3-[2-[1,3-dihydro-

275 3,3-dimethyl-1-(4-sulfobutyl)-2H-indol-2-ylidene]ethylidene]-1-cyclohexen-1-yl]ethenyl]-3,3-

276 dimethyl-1-(4-sulfobutyl)-3H-indolium inner salt sodium salt), 1,3-diphenylisobenzofuran (DPBF)

277 were purchased from Sigma-Aldrich. 1,2-distearoyl-sn-glycero-3-phosphoethanolamine-N-

278 [methoxy(polyethylene glycol)-2000] (DSPE-PEG) was obtained from Avanti Polar Lipids. TR-

279 DSPE-PEG (TR = c(RGD)-AGYLLGHINLHHLAHL(Aib)HHIL-cys) was obtained from Xi'an ruxi

280 Biological Technology. Cytiva illustra NAP-25 columns were purchased from GE Healthcare.

281 Preparation of IR783-PEG-LPs and IR783-TR-LPs

282 IR783-PEG-LPs and IR783-TR-LPs were assembled via thin film dispersion. Take the

283 preparation of IR783-TR-LPs as an example, SPC, Cho, DSPE-PEG and DSPE-PEG-TR

284 (59:33:2:6, n:n:n:n) were dissolved in mixtures of chloroform and methanol (2:1, v:v). A thin lipid

285 film was formed by evaporating with a stream of nitrogen and drying overnight under vacuum to

286 avoid residual organic solvents. Then, the thin lipid film was rehydrated with $1 \mathrm{~mL}$ of IR783

287 solution (30 mM, $10 \mathrm{mM}$ HEPES buffer, $\mathrm{pH}$ 7.4), agitation at room temperature for 30 min and

288 sonication at $-4^{\circ} \mathrm{C}$ for 5 min. Extravesicular components were removed by Sephadex G-25

289 chromatography (NAP-25 column) with 10 mM HEPES buffer, pH 7.4. Except no DSPE-PEG-TR

290 was added, the preparation of IR783-PEG-LPs was similar to the above methods.

291 Characterization of IR783-PEG-LPs and IR783-TR-LPs

292 The hydration diameter, polydispersity, and zeta potential of IR783-PEG-LPs and IR783-TR-LPs

293 were investigated using dynamic light scattering (DLS) measurements performed on NanoBrook

294 173plus equipped with a digital correlator at $659 \mathrm{~nm}$ at a scattering angle of $90^{\circ}$. The sample of

295 transmission electron microscope (TEM) was characterized by a high-resolution TEM (JEOL

296 JEM-2100F) equipped with a CCD camera. Agilent cary eclipse fluorescence spectrophotometer,

297 equipped a Cary single-cuvette peltier accessory, was used to steady-state fluorescence

298 measurements recorded in a conventional quartz cuvette (light path $10 \mathrm{~mm}$ ). A Ultraviolet-visible

299 (UV-Vis) spectrophotometer (TU-1901, Beijing Purkinje General Instrument Co.,Ltd), equipped

300 with a dual cuvette peltier accessory, was used to record UV-Vis absorbance in a quartz cuvette

301 (light path $10 \mathrm{~mm}$ ). The content of the IR783 in IR783-PEG-LPs and IR783-TR-LPs was 
calculated according to the standard curve of free IR783. The loading content and encapsulation efficiency of IR783 were determined by: $\times 100 \%$.

\section{Release of IR783 from IR783-PEG-LPs and IR783-TR-LPs at Different}

\section{pH}

309 Two phosphate buffered saline (PBS) buffers were adjusted to $\mathrm{pH} 6.5$ and $\mathrm{pH} 7.4$ by sodium 310 hydroxide and hydrochloric acid, respectively. IR783-PEG-LPs or IR783-TR-LPs was added into 311 a dialysis tubing (3.5K MWCO), which was carefully placed in a beaker $(50 \mathrm{~mL})$ containing above 312 PBS buffer $(20 \mathrm{~mL})$. The temperature, stirring speed and working time of the heating plate were 313 set to $37^{\circ} \mathrm{C}, 200 \mathrm{rpm}$ and $48 \mathrm{~h}$, respectively. A sample $(500 \mu \mathrm{L})$ was taken from the dialysate at 314 regular intervals to measure the absorption spectrum. Finally, the released IR783 was measured 315 by UV/vis absorption spectra according to the standard curve. After each sample, fresh PBS 316 buffer $(500 \mu \mathrm{L})$ was immediately added into the beaker to restore the original volume. Thus, the 317 cumulative percentage of the released IR783 was calculated by:

318 The cumulative percentage of the released IR783 $(\%)=($ Cumulative weight of released 319 IR783 in dissolution medium)/Total weight of IR783 in IR783-PEG-LPs or IR783-TR-LPs) $\times 100 \%$.

\section{$320 \quad$ ROS generation measurements of IR783}

321 The production of ROS from IR783 was monitored using DPBF as an ROS indicator based on 322 degradation of DPBF, the absorption at $410 \mathrm{~nm}$ of DPBF reduced. In this experiment, a NIR laser 323 source $(\lambda=808 \mathrm{~nm})$ was performed at room temperature to examine the photophysical ability of 324 IR783. As a control experiment, DPBF $(50 \mu \mathrm{M})$ in dimethylformamide (DMF) absence IR783 was 325 irradiated by a same NIR laser source. For DPBF itself, the absorbance of DPBF at $410 \mathrm{~nm}$ had 326 no reduction observed in experimental time of irradiation, which indicated DPBF was stable under 327 experimental conditions. Then, we examined the ROS generation of IR783 (10 $\mu \mathrm{M})$ using DPBF $328(50 \mu \mathrm{M})$ in $2.0 \mathrm{~mL}$ of DMF. Upon irradiation $(\lambda=808 \mathrm{~nm})$, the absorbance at $\lambda=410 \mathrm{~nm}$ was 
detected by UV-Vis spectroscopy within 100 seconds. Furthermore, vitamin C (VC), a ROS scavenger, was used to verify ROS production of IR783. Addition of VC (100 mM) with IR783 (10 $\mu \mathrm{M})$ and DPBF $(50 \mu \mathrm{M})$ in $2.0 \mathrm{~mL}$ of DMF, the absorbance at $\lambda=410 \mathrm{~nm}$ was detected by UV-Vis

332 spectroscopy within 100 seconds.

\section{Photothermal properties of IR783-PEG-LPs and IR783-TR-LPs}

334 The photothermal properties of IR783-PEG-LPs and IR783-TR-LPs and free IR783 aqueous

335 solution under laser irradiation in Eppendorf tubes were determined with a thermal imaging

336 camera (Fotric 225S). IR783-PEG-LPs and IR783-TR-LPs and free IR783 at different

337 concentrations $(0,0.25,0.5,1.0 \mathrm{mM}$ of IR783, $0.2 \mathrm{~mL})$ were put into Eppendorf tubes and

338 irradiated by the NIR laser $\left(808 \mathrm{~nm}, 2 \mathrm{~W} / \mathrm{cm}^{2}\right)$. Moreover, IR783-PEG-LPs and IR783-TR-LPs

339 and free IR783 at fixed concentration of IR783 $(0.5 \mathrm{mM}, 0.2 \mathrm{~mL})$ was put into Eppendorf tubes

340 and irradiated by $808 \mathrm{~nm}$ laser $\left(0,1,2,3 \mathrm{~W} / \mathrm{cm}^{2}\right)$. The temperature was automatically recorded

341 every 30 seconds for $10 \mathrm{~min}$.

\section{Intracellular ROS detection}

343 The generation of intracellular ROS was measured by laser confocal microscope (CLSM) using 3442 2',7'-Dichlorodihydrofluorescein diacetate (DCFH-DA) as the ROS probe. After treating 4T1 cells 345 with the IR783-PEG-LPs, IR783-TR-LPs and free IR783 (10 $\mu \mathrm{M}$, the concentration of IR783) for 6

$346 \mathrm{~h}$ in dark, the previous medium were replaced with fresh medium. DCFH-DA was added (final 347 concentration of $10 \mu \mathrm{M}$ ) and incubate for $30 \mathrm{~min}$. Subsequently, the cells were washed with PBS 348 three times and further illuminated with a NIR laser $\left(808 \mathrm{~nm}, 1 \mathrm{~W} / \mathrm{cm}^{2}\right)$ for $2 \mathrm{~min}$, followed by 349 imaging with CLSM. The excitation light was selected to be $488 \mathrm{~nm}$ and the emission spectra in 350 the wavelength range of $510-540 \mathrm{~nm}$ were collected by CLSM. Alternatively, before treatment 351 with the IR783-PEG-LPs, IR783-TR-LPs and free IR783, the cells were pre-treated with N352 acetylcysteine (1.0 mM) for $2 \mathrm{~h}$. In addition, the IR783-PEG-LPs, IR783-TR-LPs and free IR783 353 nanoparticle-treated cells without laser irradiation, the cells with only laser irradiation, and the 354 cells without any treatment were used as controls.

\section{Intracellular PTT and PDT}


Cell culture: Murine 4T1 breast cancer cells were incubated in DMEM medium with 10\% FBS and $1 \%$ penicillin streptomycin at $37^{\circ} \mathrm{C}$ in a humidified atmosphere containing $5 \% \mathrm{CO}_{2}$. Before experiments, the cells were pre-cultured until confluence was reached.

The cell viability of the IR783-PEG-LPs, IR783-TR-LPs and free IR783 against 4T1 cancer cells was evaluated by 3-(4,5-dimethylthiazol-2-yl)-2,5-diphenyl tetrazolium bromide (MTT) assay. In brief, 4T1 cells were seeded in 96-well plates at an appropriate density of $5 \times 10^{3}$ cells per well, respectively. After adherence, the 4T1 cancer cells were treated with IR783-PEG-LPs, IR783-TRLPs and free IR783 (10 $\mu \mathrm{M}$, the concentration of IR783) in the dark. After 6 hours of incubation, the previous medium was replaced with fresh medium. The cells were irradiated with a NIR laser (808 $\mathrm{nm}, 0.1$ and $\left.0.5 \mathrm{~W} / \mathrm{cm}^{2}\right)$ for $2 \mathrm{~min}$. Then, $100 \mu \mathrm{L}$ of MTT solution $(0.5 \mathrm{mg} / \mathrm{mL}$ ) was added to each well and incubated for $4 \mathrm{~h}$. Thereafter, the medium was removed and $100 \mu \mathrm{L}$ of DMSO was added, followed by gently shaking for $10 \mathrm{~min}$. The absorbance was measured at $490 \mathrm{~nm}$ using a microplate reader (Bio-Rad, iMark, USA). The relative cell viability was calculated as: cell viability $=(\mathrm{OD} 490($ samples$) / \mathrm{OD} 490($ control $)) \times 100 \%$, where OD490 (control) was obtained in the absence of IR783-PEG-LPs, IR783-TR-LPs and free IR783, and OD490 (samples) was obtained in the presence of IR783-PEG-LPs, IR783-TR-LPs and free IR783. Each value was averaged from three independent experiments. As the control, the cytotoxicity of IR783-PEG-LPs, IR783TR-LPs and free IR783 in the dark was also evaluated. Each value was averaged from three 374 independent experiments.

\section{In vivo imaging and antitumor study}

376 In terms of animal and tumor models, all animal research were conducted in accordance with the 377 guidelines established by the Guizhou Provincial Science Committee, and the entire project 378 protocol was approved by the Animal Ethics Committee of Zunyi Medical University. Female 379 BALB/c mice (5-6 weeks old) were bought from Vital River Laboratory Animal Technology 380 (Beijing, China). The mice were used for in vivo fluorescence imaging and anti-tumor 381 experiments. To establish the xenograft $4 \mathrm{~T} 1$ tumor-bearing mouse model, $1 \times 10^{7} 4 \mathrm{~T} 1$ cancer cells 382 were injected subcutaneously into the right side of the mouse. The mice with tumor volumes at 383 around $100 \mathrm{~mm}^{3}$ were used subsequently. 
In vivo fluorescence imaging: The 4T1 tumor-bearing mice were intravenously administrated with $100 \mu \mathrm{L}$ of IR783-PEG-LPs, IR783-TR-LPs and free IR783 solution (containing IR783: 1 $\left.\mathrm{mg} \cdot \mathrm{kg}^{-1}\right)$. Then the mice were anesthetized and imaged via a IVIS Lumina XRMS Series III system (PerkinEImer, MA, USA) at 0, 2, 4, 824 and $48 \mathrm{~h}$ post-injection. The excitation wavelength was $720 \mathrm{~nm}$ and in vivo spectral imaging from 790 to $850 \mathrm{~nm}$ (with $10 \mathrm{~nm}$ steps) was carried out. For the ex vivo tissues distribution study, the mice were sacrificed after $48 \mathrm{~h}$ injection, and tumor as well as major organs (heart, liver, spleen, lung, and kidney) were collected and subjected for ex vivo imaging.

In vivo photothermal imaging: To directly evaluate the photothermal effect of IR783-PEGLPs, IR783-TR-LPs and free IR783 in vivo, Thermal imaging was applied to detect temperature changes in mice under NIR laser irradiation. The tumor-bearing mice were randomly divided into four groups of five mice each, and intravenously injected with IR783-PEG-LPs, IR783-TR-LPs and free IR783. The dose was adjusted at $1 \mathrm{mg} / \mathrm{kg}$ for IR783. As a control, mice were given the same volume of saline. The tumor-bearing mice treated with IR783-PEG-LPs, IR783-TR-LPs and free IR783 were irradiated with a NIR laser $\left(808 \mathrm{~nm}, 0.5 \mathrm{~W} / \mathrm{cm}^{2}\right)$ at $8 \mathrm{~h}$ post injection. Temperature changes and the IR images were acquired at 0, 1, 3, and $5 \mathrm{~min}$.

For antitumor study, the tumor-bearing mice were randomly divided into four groups of five mice each: 1) "Saline", 2) "IR 783", 3) " IR783-PEG-LPs" 4) ", IR783-TR-LPs". The animals were 402 intravenously administrated with saline, free IR $783\left(1 \mathrm{mg} \cdot \mathrm{kg}^{-1}\right)$, IR783-PEG-LPs and IR783-TR403 LPs (at dose of IR783 $1 \mathrm{mg} \cdot \mathrm{kg}^{-1}$ ), respectively, via the tail vein on day 0 . Then, the mice were 404 irradiated with a NIR laser $\left(808 \mathrm{~nm}, 500 \mathrm{~mW} / \mathrm{cm}^{2}\right)$ for $5 \mathrm{~min}$ at $8 \mathrm{~h}$. The tumor size and body 405 weight of the mice were measured every three days for 15 days. Tumor size was measured by a 406 caliper and tumor volume was calculated using the following formula: $V=W^{2} \times L / 2$, where $W$ and $407 \quad \mathrm{~L}$ were the shortest and longest diameters of tumors, respectively. Relative tumor size was 408 calculated as $\mathrm{V} / \mathrm{V}_{0}\left(\mathrm{~V}_{0}\right.$ was the initial tumor volume). On day 15 , all mice were sacrificed and the 409 tumors were excised. 


\section{Acknowledgments}

411 Not applicable.

\section{Authors' contributions}

413 ZLY and JG designed the experimental protocols. JJL carried out all the studies with help from

414 TJL, MYY and MMW. JJL wrote the manuscript with help from ZLY and JG.

\section{Funding}

416 This work was supported by the Natural Science Foundation of China (Grant No. 82060626,

417 81360471), Innovative Group Project of Guizhou Province of Education (KY[2018]024), Guizhou

418 Science and Technology Support Program([2020]4Y158), Talents of Guizhou Science and

419 Technology Cooperation Platform [2020]4104. Cultivation project plan of new seedling cultivation

420 and innovation exploration special project of Zunyi Medical University, Platform talents of

421 Qiankehe, Grant/Award Number: [2018] No. 5772-030.

422 Availability of data and materials

423 All data generated or analyzed in this study are included in this article and its attached files.

\section{Ethics approval and consent to participate}

425 The use of animals was approved by and in accordance with the animal welfare committee of

426 Zunyi Medical University.

\section{Consent for publication}

428 All authors agree to the publication of this study. 


\section{Competing interests}

430 The author reports no conflicts of interest in this work.

\section{Author details}

$432{ }^{1}$ Key Laboratory of Basic Pharmacology of Ministry of Education and Joint International Research

433 Laboratory of Ethnomedicine of Ministry of Education, Zunyi Medical University, Zunyi, Guizhou, 434563003 , China.

435 2Key Laboratory of Biocatalysis \& Chiral Drug Synthesis of Guizhou Province, Zunyi, Guizhou 436563003, P. R. China.

437 35chool of Pharmacy, Zunyi Medical University, Zunyi, Guizhou 563003, P. R. China.

$438{ }^{4}$ Guizhou International Scientific and Technological Cooperation Base for Medical Photo-

439 Theranostics Technology and Innovative Drug Development, Zunyi, Guizhou 563003, P. R.

440 China.

442 Correspondence: Jie Gao and Ze-li Yuan

443 School of Pharmacy, Key Laboratory of Basic Pharmacology of Ministry of Education and Joint

444 International Research Laboratory of Ethnomedicine of Ministry of Education Zunyi Medical

$445 \quad$ University

$446 \quad$ No.6 West Xuefu Road, Zunyi, Guizhou 563003, P. R. China

447 Tel:+86-851-286-423-36

448 Fax:+86-851-286-432-36

449 E-mail: jiegao@mail.nankai.edu.cn; zlyuan@zmu.edu.cn

450

451 References

452 1. Luo DD, Carter KA, Miranda D, Lovell JF. Chemophototherapy: An Emerging Treatment $453 \quad$ Option for Solid Tumors. Adv Sci 2017. 
2. Li X, Lee S, Yoon J. Supramolecular photosensitizers rejuvenate photodynamic therapy. Chem Rev. 2018;47:1174-88.

3. Li JC, Rao JH, Pu KY. Recent progress on semiconducting polymer nanoparticles for molecular imaging and cancer phototherapy. Biomaterials. 2018;155:217-35.

4. Gai SL, Yang GX, Yang PP, He F, Lin J, Jin DY, Xing BG. Recent advances in functional nanomaterials for light-triggered cancer therapy. Nano Today. 2018;19:146-87.

5. Sun H, Zhang Q, Li J, Peng S, Wang X, Cai R. Near-infrared photoactivated nanomedicines for photothermal synergistic cancer therapy. Nano Today. 2021;37:101073.

6. Fan W, Yung B, Huang P, Chen X. Nanotechnology for Multimodal Synergistic Cancer Therapy. Chem Rev. 2017;117:13566-638.

7. Gao J, Li J, Geng W-C, Chen F-Y, Duan X, Zheng Z, et al. Biomarker Displacement Activation: A General Host-Guest Strategy for Targeted Phototheranostics in Vivo. J Am Chem Soc. 2018;140:4945-53.

8. Liu YJ, Bhattarai P, Dai ZF, Chen XY. Photothermal therapy and photoacoustic imaging via nanotheranostics in fighting cancer. Chem Rev. 2019;48:2053-108.

9. Jung HS, Verwilst P, Sharma A, Shin J, Sessler JL, Kim JS. Organic molecule-based photothermal agents: an expanding photothermal therapy universe. Chem Soc Rev. 2018;47:2280-97.

10. Hong G, Antaris AL, Dai H. Near-infrared fluorophores for biomedical imaging. Nat Biomed Eng 2017.

11. Yoon H-J, Lee H-S, Lim J-Y, Park J-H. Liposomal Indocyanine Green for Enhanced Photothermal Therapy. ACS Appl Mater Interfaces. 2017;9:5683-91.

12. Zhang L, Yi H, Song J, Huang J, Yang K, Tan B, et al. Mitochondria-Targeted and Ultrasound-Activated Nanodroplets for Enhanced Deep-Penetration Sonodynamic Cancer Therapy. ACS Appl Mater Interfaces. 2019;11:9355-66.

13. Lim W, Jo G, Lee BY, Park MH, Hyun H. Rapid Clearance of IR783 and Methyl- $\beta$ cyclodextrin Complex for Improved Tumor Imaging. Part. Part. Syst. Charact. 2021;38:2100068. 
14. Peer D, Karp JM, Hong S, Farokhzad OC, Margalit R, Langer R. Nanocarriers as an emerging platform for cancer therapy. Nat Nanotechnol. 2007;2:751-60.

15. Ruoslahti E, Pierschbacher MD. New perspectives in cell adhesion: RGD and integrins. Science. 1987;238:491-7.

16. Danhier F, Le Breton A, Préat V. RGD-based strategies to target alpha(v) beta(3) integrin in cancer therapy and diagnosis. Mol Pharmaceutics. 2012;9:2961-73.

17. Shi K, Long $Y, X u C$, Wang $Y$, Qiu $Y, Y u$ Q, et al. Liposomes Combined an Integrin avß3Specific Vector with pH-Responsible Cell-Penetrating Property for Highly Effective Antiglioma

20. Barua S, Mitragotri S. Challenges associated with Penetration of Nanoparticles across Cell and Tissue Barriers: A Review of Current Status and Future Prospects. Nano Today. 2014;9:223-43.

21. Li C, Greenwood TR, Bhujwalla ZM, Glunde K. Synthesis and characterization of glucosamine-bound near-infrared probes for optical imaging. Org Lett. 2006;8:3623-6. 


\begin{tabular}{lllllll}
\hline Samples & $\mathbf{p H}$ & $\begin{array}{l}\text { Diameter } \\
(\mathbf{n m})\end{array}$ & Zeta potential $(\mathbf{m V})$ & PDI & EE (\%) & DL (\%) \\
& & & & & \\
\hline TR-LPS & 6.5 & $104.4 \pm 2.0$ & $+5.09 \pm 0.46$ & $0.205 \pm 0.023$ & - & - \\
& 7.4 & $143.3 \pm 1.1$ & $-7.83 \pm 2.19$ & $0.215 \pm 0.027$ & $19.0 \pm 2.6$ & $11.2 \pm 1.5$ \\
& 6.5 & $102.3 \pm 1.0$ & $-18.15 \pm 4.48$ & $0.209 \pm 0.011$ & - & - \\
PEG-LPS & 6.4 & $104.0 \pm 1.5$ & $-16.00 \pm 0.84$ & $0.163 \pm 0.003$ & $21.8 \pm 1.5$ & $14.8 \pm 2.6$ \\
& & & & & & \\
\hline
\end{tabular}

506 Scheme 1 Schematic structure of IR783-TR-LPs.

507 Figure 1 Characteristics of TR-LPs. Size distribution (A) and TEM image of TR-LPs. Scale bar 508 was $500 \mathrm{~nm}$. (C) Size of TR-LPs changes with time in PBS buffer (pH 7.4), DMEM and 10\% FBS. 509 (D) UV-vis spectra and images of free IR783, IR783-PEG LPs and IR783-TR-LPs. (E) Photo510 stability of free IR783, IR783-PEG LPs and IR783-TR-LPs under irradiation of LED light (white 511 light, $6.78 \mathrm{~W}$ ) at room temperature. (F) The cumulative percentage of IR783 released from IR783512 TR-LPs at pH 6.5 and 7.4 (PBS buffer, $10 \mathrm{mM}, 37^{\circ} \mathrm{C}$ ).

513 Figure 2 Phototheranostic properties of IR783-LPs. The photothermal properties of IR783-TR-

514 LPs in PBS buffer at fixed IR783 concentration $(0.5 \mathrm{mM})$ exposed to laser irradiation $(0,1,2,3$

$\left.515 \mathrm{~W} / \mathrm{cm}^{2}\right)(\mathrm{A})$ or at different IR783 concentration $(0,0.25,0.5,1 \mathrm{mM})$ exposed to NIR laser light $516\left(808 \mathrm{~nm}, 2 \mathrm{~W} / \mathrm{cm}^{2}\right)$ (B) for $10 \mathrm{~min}$. Photothermal imaging (C) and photothermal cycles (D) of 517 IR783-TR-LPs. (E) The ROS generation of free IR783.

518 Figure $3 \mathrm{In}$ vitro phototherapy evaluation. (A) Intracellular ${ }^{1} \mathrm{O}_{2}$ detection by CLSM after $4 \mathrm{~T} 1$ cells 519 were treated with the IR783-TR-LPs, IR783-PEG-LPs, free IR783 and DCFH-DA with $808 \mathrm{~nm}$ 520 laser irradiation (1), no irradiation (2) and addition of $\mathrm{N}$-acetylcysteine with $808 \mathrm{~nm}$ laser 521 irradiation (3). Cells treated with DCFH-DA under irradiation (4) or dark (5) as control. Scale bar, $52250 \mu \mathrm{m}$. (B) The cell viability of $4 \mathrm{~T} 1$ cells were treated with the IR783-TR-LPs, IR783-PEG-LPs 523 and free IR783 under different power of laser irradiation at pH 7.4. (C) The cell viability of 4T1 
524 cells were treated with the IR783-TR-LPs, IR783-PEG-LPs and free IR783 under different power 525 of laser irradiation at $\mathrm{pH} 6.5$.

526 Figure 4 In vivo fluorescence and photothermal imaging. (A) In vivo fluorescence imaging of the 5274 T1 tumor-bearing mice at 2, 4, 8, 24 and $48 \mathrm{~h}$ after i.v. of IR783-TR-LPs, IR783-PEG-LPs and 528 free IR783. Red cycles indicate the tumor sites. Ex vivo imaging of tumor and major organs 529 harvested from the euthanized $4 \mathrm{~T} 1$ tumor-bearing nude mice at $8 \mathrm{~h}$ post-injection. (B) In vivo 530 photothermal imaging of the $4 T 1$ tumor-bearing mice under laser irradiation $\left(808 \mathrm{~nm}, 0.5 \mathrm{~W} / \mathrm{cm}^{2}\right)$ 531 at 1, 3 and $5 \mathrm{~min}$ (eight hours after intravenous injection).

532 Figure 5 In vivo PDT/PTT tumor ablation. (A) Changes of tumor volume in four groups. (B)

533 Photos of the excised tumor. (C) Changes of body weight in four groups. (D) Histological sections 534 of tumor tissues after treatment were stained with TUNEL and H\&E, respectively. All scale bars 535 are $100 \mu \mathrm{m}$. Error bars are based on standard error of mean $\left({ }^{* *} p<0.05\right)$.

536 Figure 6 IR783 encapsulated in TR-conjugated liposomes for enhancing NIR imaging-guided $537 \quad$ PTT and PDT 
Figures
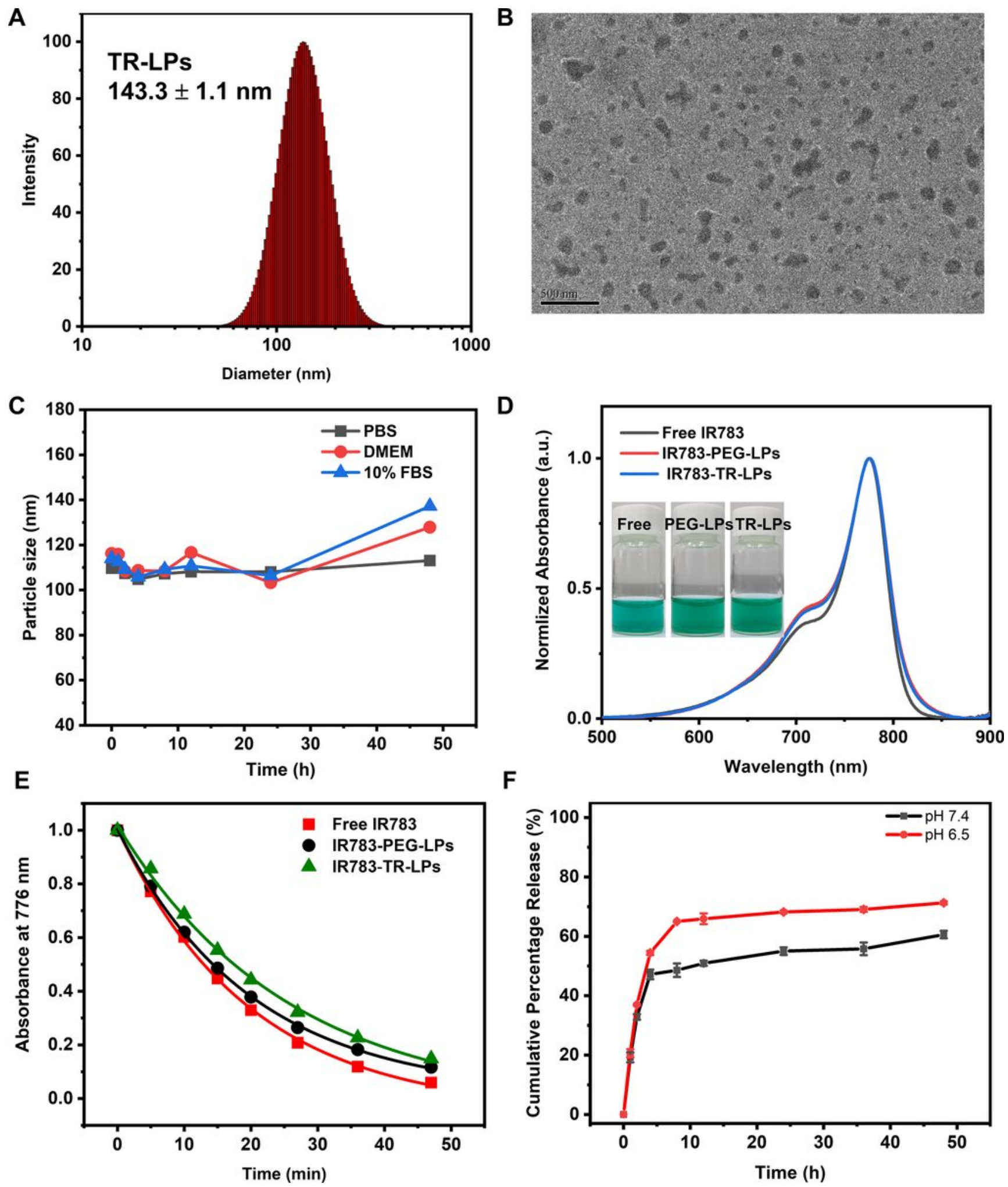

$\mathbf{F}$

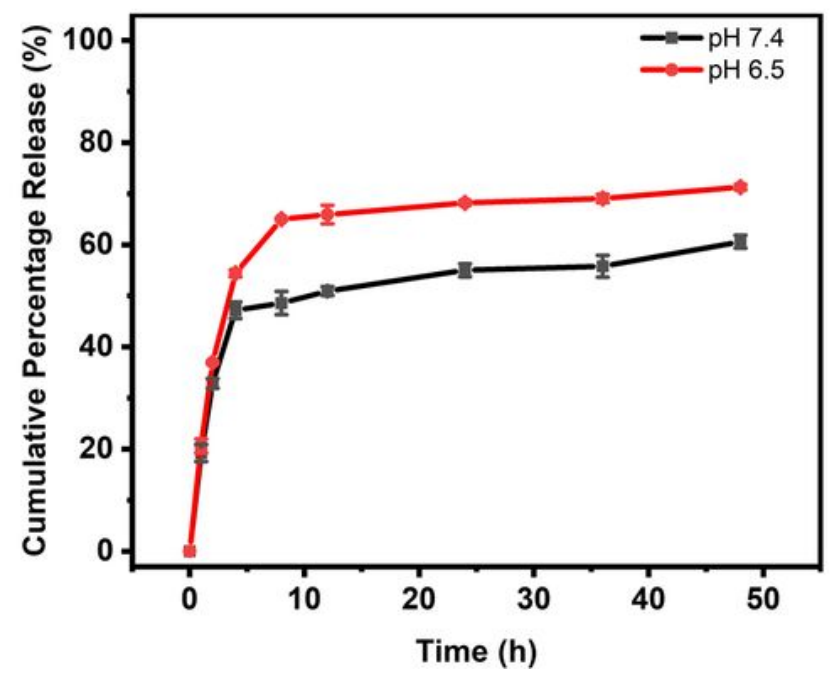

Figure 1

Characteristics of TR-LPs. Size distribution (A) and TEM image of TR-LPs. Scale bar was $500 \mathrm{~nm}$. (C) Size of TR-LPs changes with time in PBS buffer (pH 7.4), DMEM and 10\% FBS. (D) UV-vis spectra and images of free IR783, IR783-PEG LPs and IR783-TR-LPs. (E) Photo-stability of free IR783, IR783-PEG LPs 
and IR783-TR-LPs under irradiation of LED light (white light, $6.78 \mathrm{~W}$ ) at room temperature. (F) The cumulative percentage of IR783 released from IR783-TR-LPs at pH 6.5 and 7.4 (PBS buffer, 10 mM, 37 $\left.{ }^{\circ} \mathrm{C}\right)$.

A

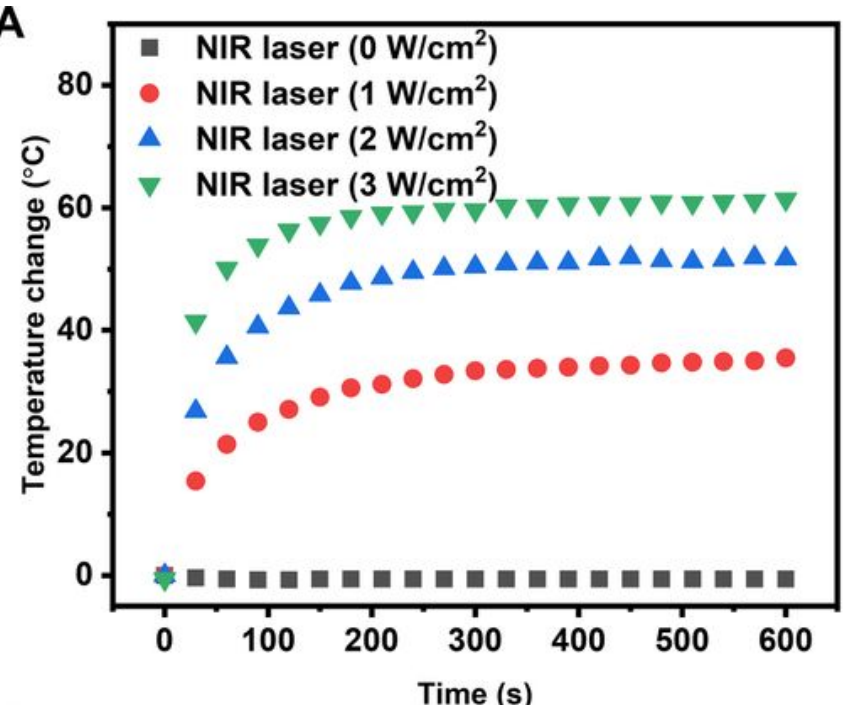

C

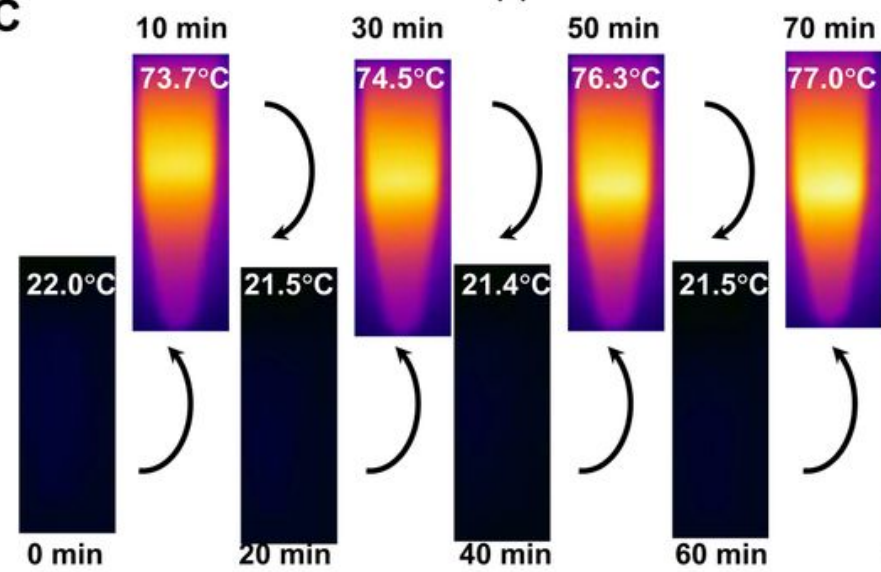

B
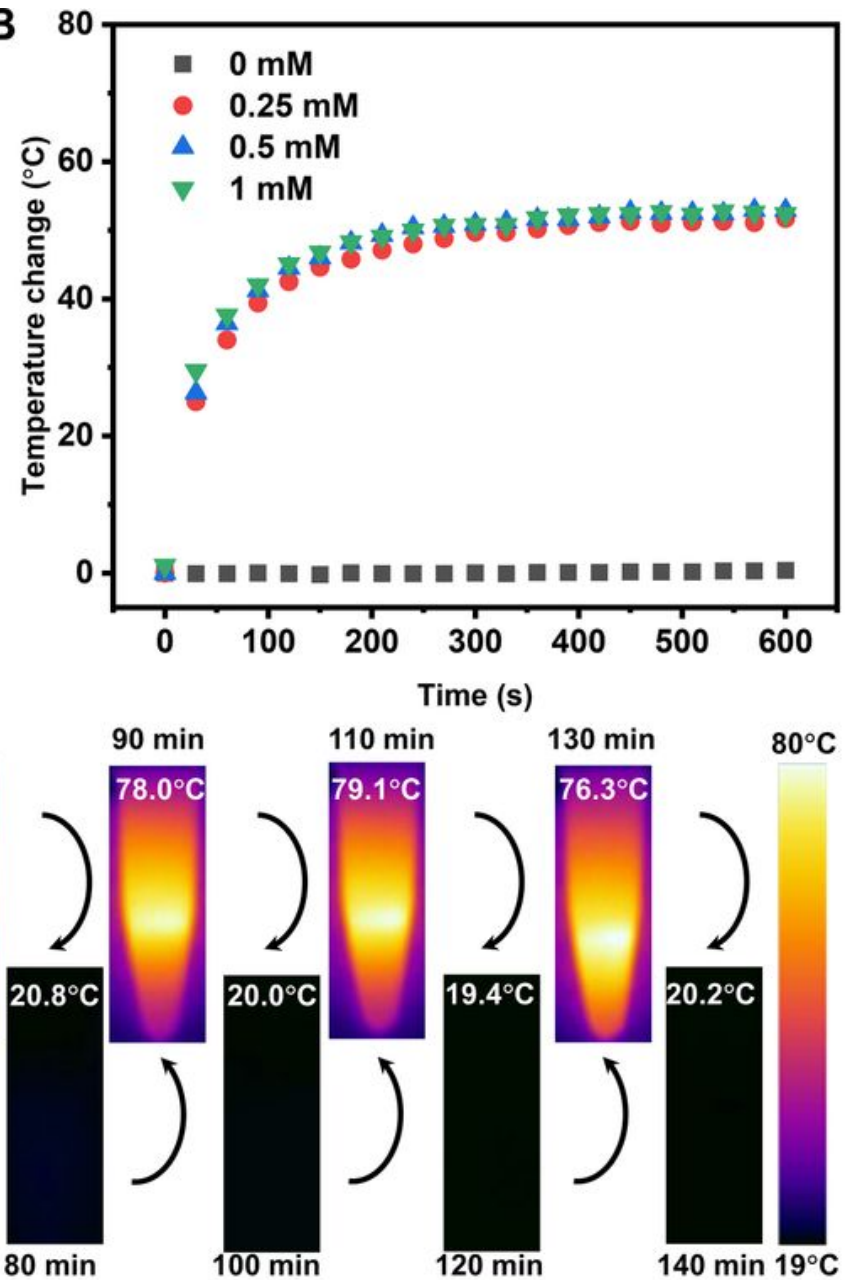
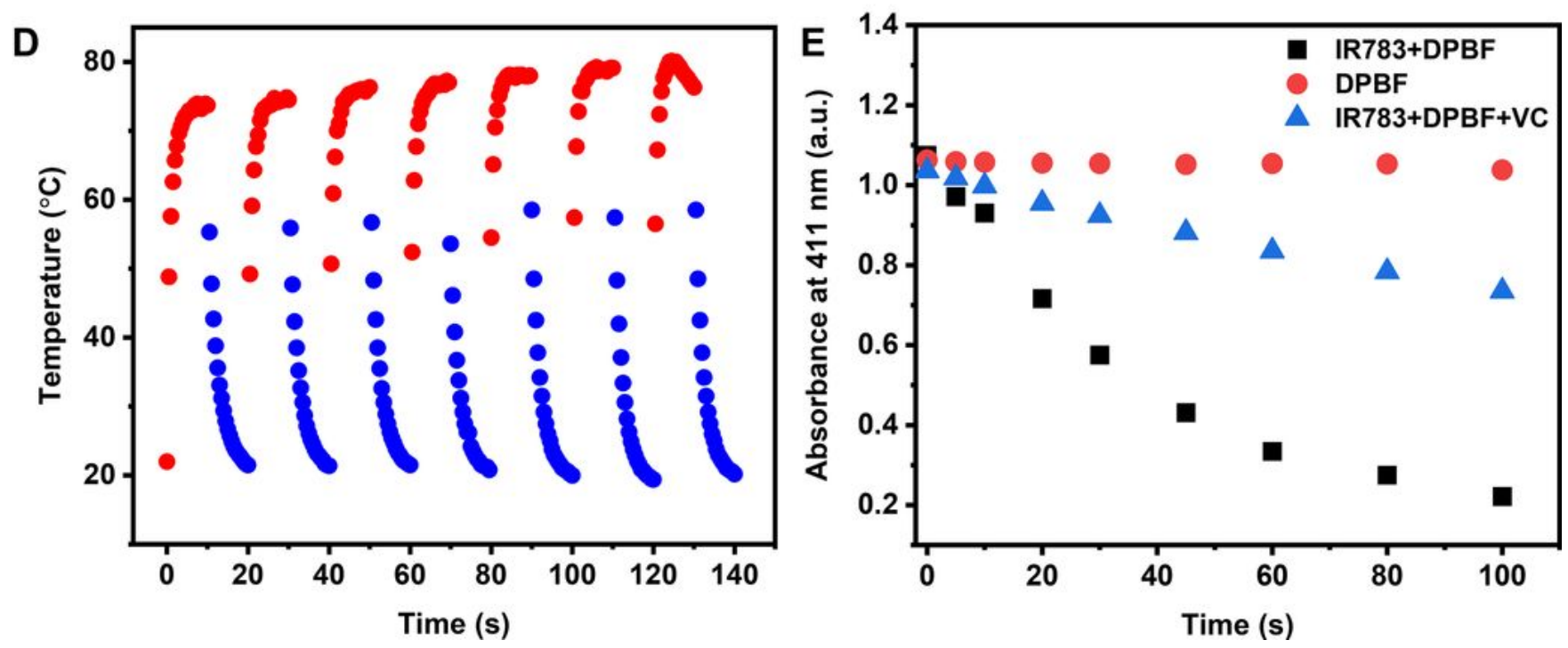

Figure 2

Phototheranostic properties of IR783-LPS. The photothermal properties of IR783-TR-LPs in PBS buffer at fixed IR783 concentration $(0.5 \mathrm{mM})$ exposed to laser irradiation $(0,1,2,3 \mathrm{~W} / \mathrm{cm} 2)(\mathrm{A})$ or at different IR783 
concentration $(0,0.25,0.5,1 \mathrm{mM})$ exposed to NIR laser light (808 nm, $2 \mathrm{~W} / \mathrm{cm} 2)(B)$ for $10 \mathrm{~min}$.

Photothermal imaging (C) and photothermal cycles (D) of IR783-TR-LPS. (E) The ROS generation of free IR783.

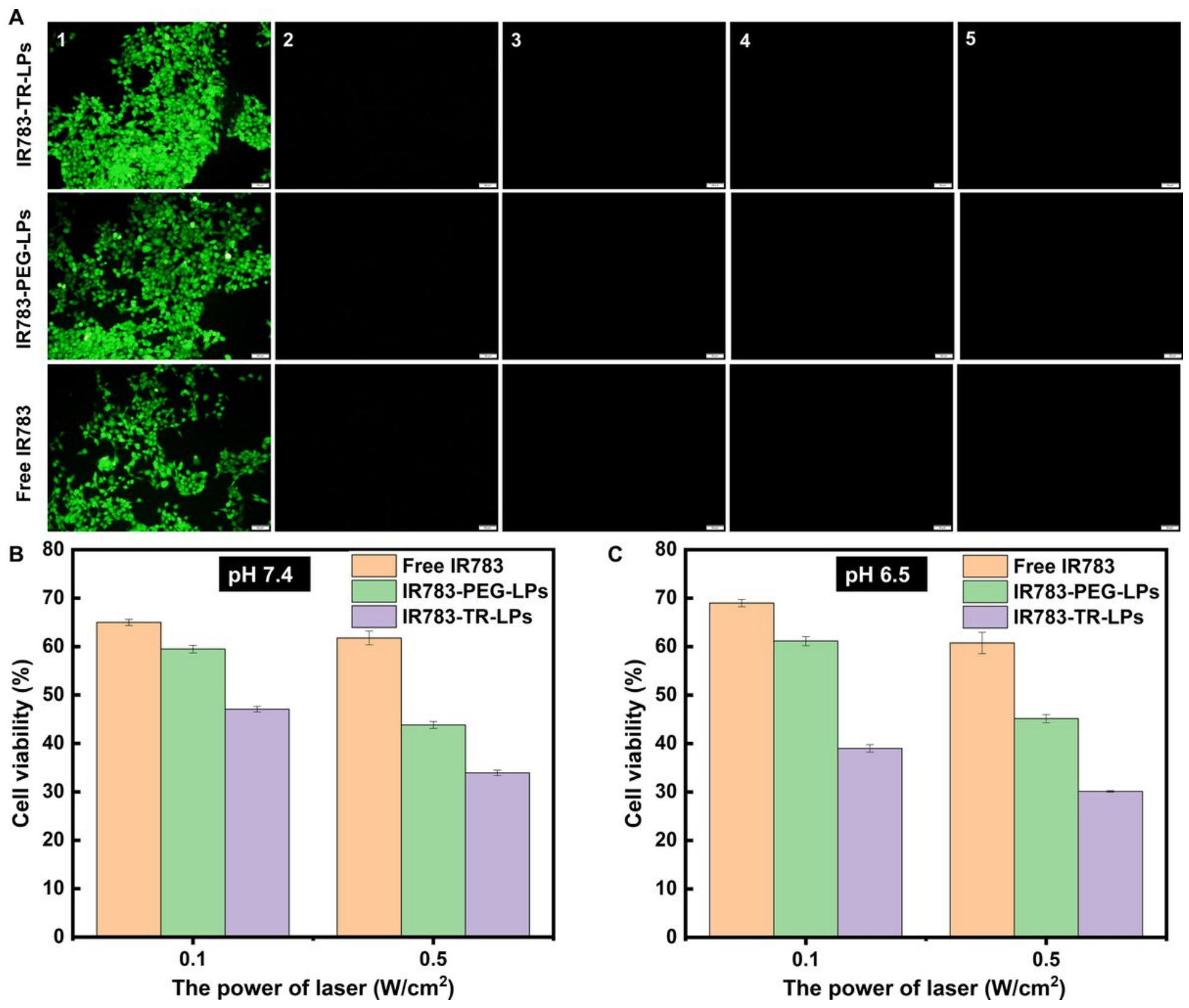

Figure 3

In vitro phototherapy evaluation. (A) Intracellular 102 detection by CLSM after 4T1 cells were treated with the IR783-TR-LPS, IR783-PEG-LPs, free IR783 and DCFH-DA with $808 \mathrm{~nm}$ laser irradiation (1), no irradiation (2) and addition of N-acetylcysteine with $808 \mathrm{~nm}$ laser irradiation (3). Cells treated with DCFHDA under irradiation (4) or dark (5) as control. Scale bar, $50 \mu \mathrm{m}$. (B) The cell viability of 4T1 cells were treated with the IR783-TR-LPs, IR783-PEG-LPs and free IR783 under different power of laser irradiation at $\mathrm{pH}$ 7.4. (C) The cell viability of $4 T 1$ cells were treated with the IR783-TR-LPS, IR783-PEG-LPs and free IR783 under different power of laser irradiation at $\mathrm{pH} 6.5$. 
A
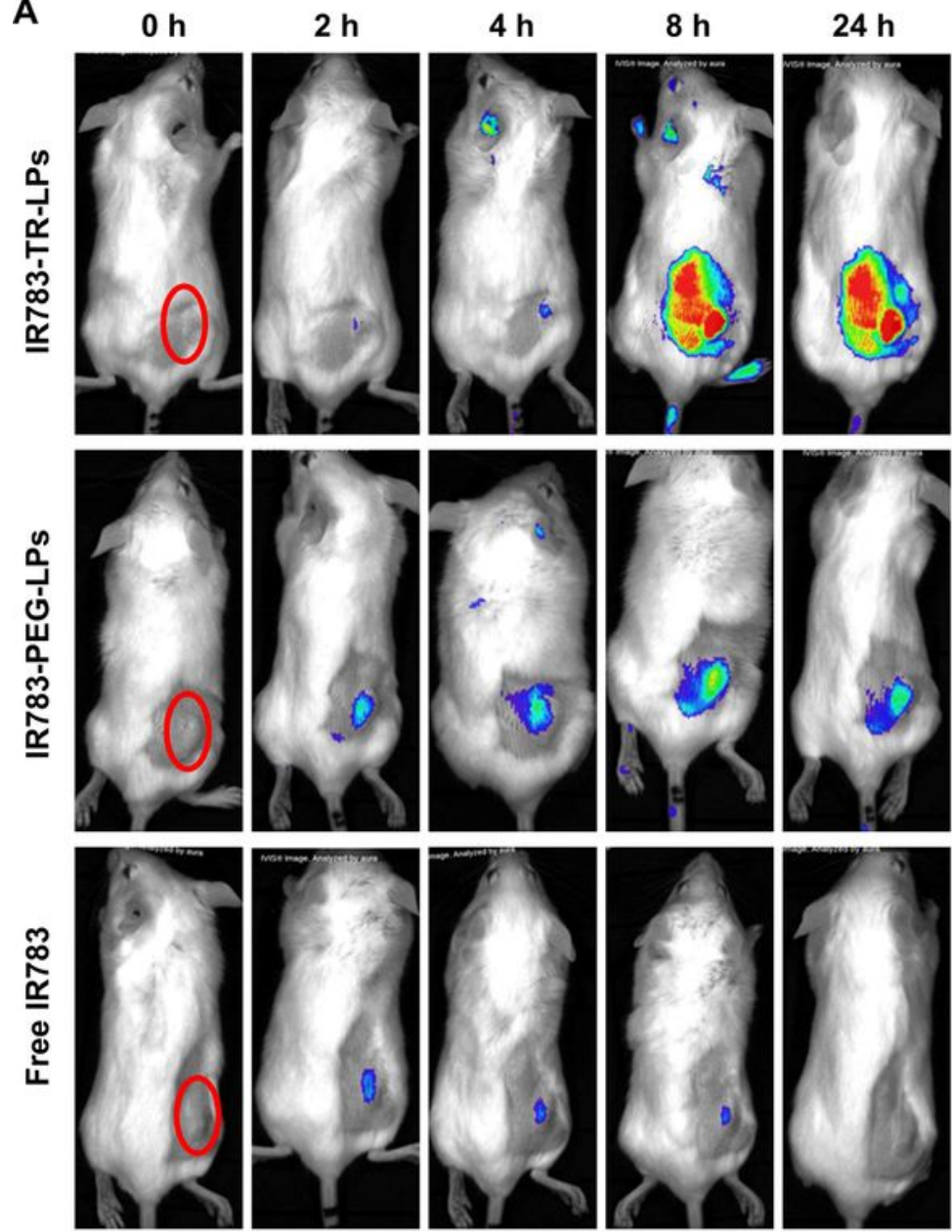

B
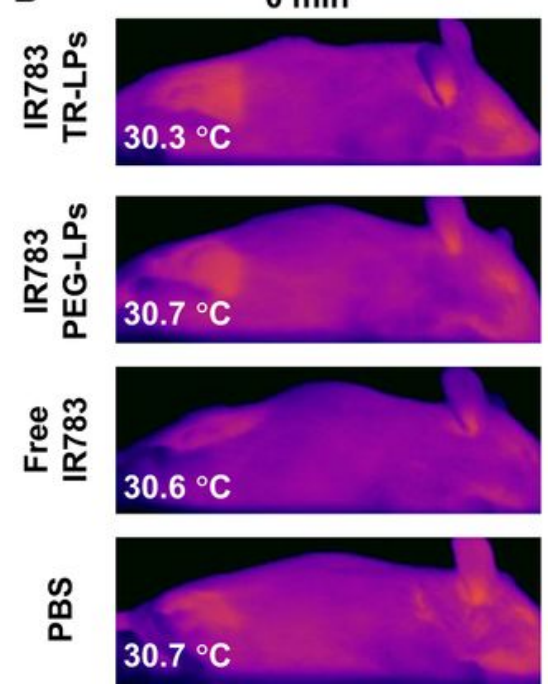
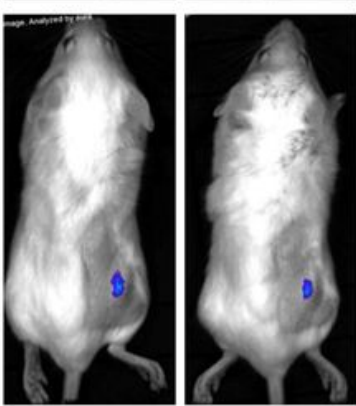

$1 \mathrm{~min}$
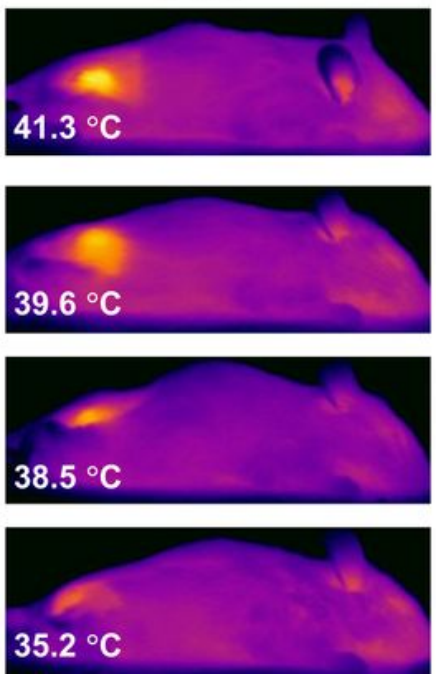

$48 \mathrm{~h}$
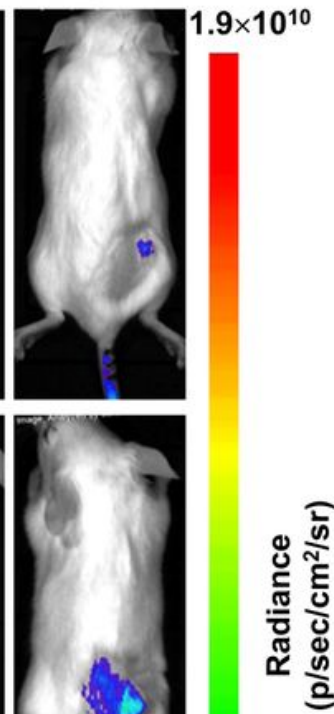

$7.6 \times 10^{9}$

$3 \mathrm{~min}$
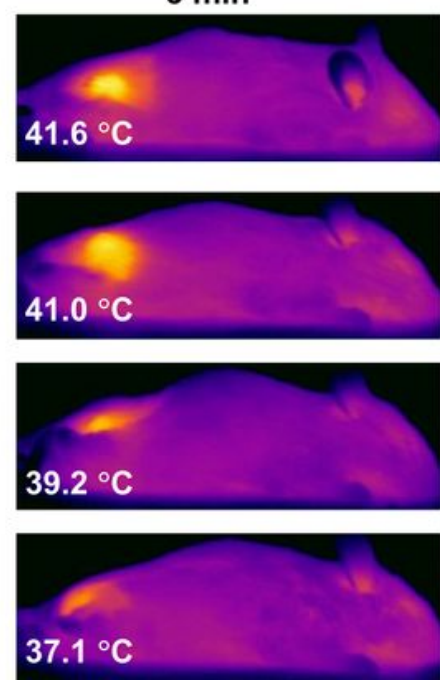

(.)

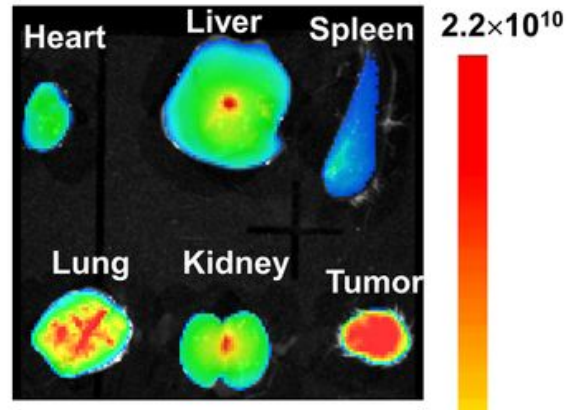

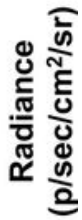
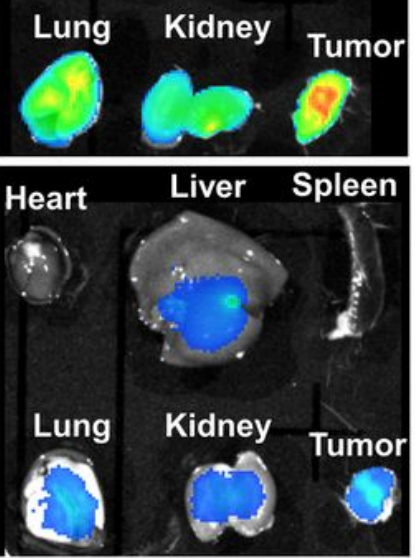

$1.8 \times 10^{8}$

$5 \mathrm{~min}$
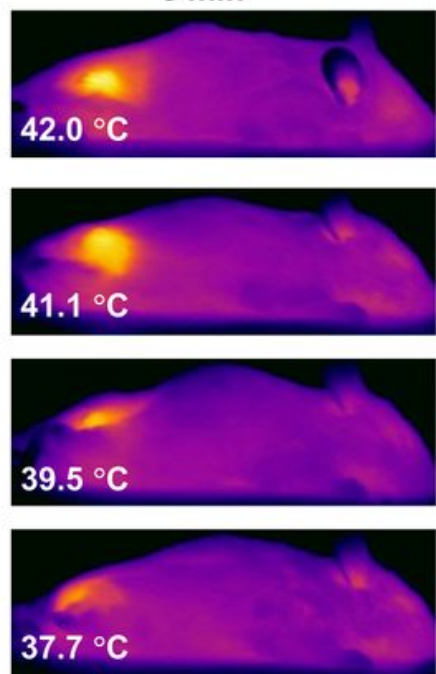

Figure 4

In vivo fluorescence and photothermal imaging. (A) In vivo fluorescence imaging of the 4T1 tumorbearing mice at 2, 4, 8, 24 and $48 \mathrm{~h}$ after i.v. of IR783-TR-LPs, IR783-PEG-LPs and free IR783. Red cycles indicate the tumor sites. Ex vivo imaging of tumor and major organs harvested from the euthanized 4T1 tumor-bearing nude mice at $8 \mathrm{~h}$ post-injection. (B) In vivo photothermal imaging of the $4 \mathrm{~T} 1$ tumor-bearing 
mice under laser irradiation $(808 \mathrm{~nm}, 0.5 \mathrm{~W} / \mathrm{cm} 2)$ at 1, 3 and $5 \mathrm{~min}$ (eight hours after intravenous injection).
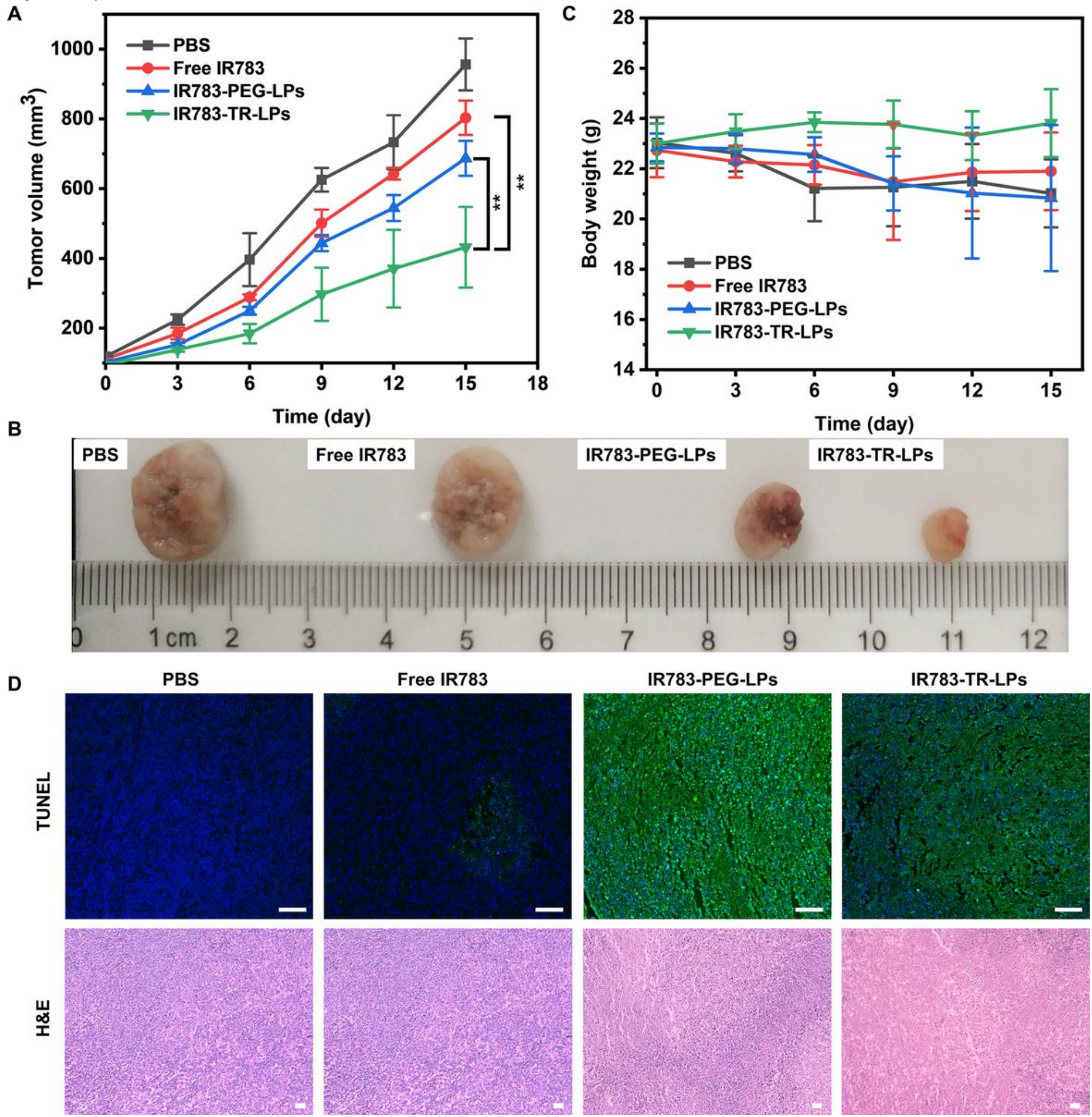

Figure 5

In vivo PDT/PTT tumor ablation. (A) Changes of tumor volume in four groups. (B) Photos of the excised tumor. (C) Changes of body weight in four groups. (D) Histological sections of tumor tissues after 
treatment were stained with TUNEL and H\&E, respectively. All scale bars are $100 \mu \mathrm{m}$. Error bars are based on standard error of mean $(* \star p<0.05)$.

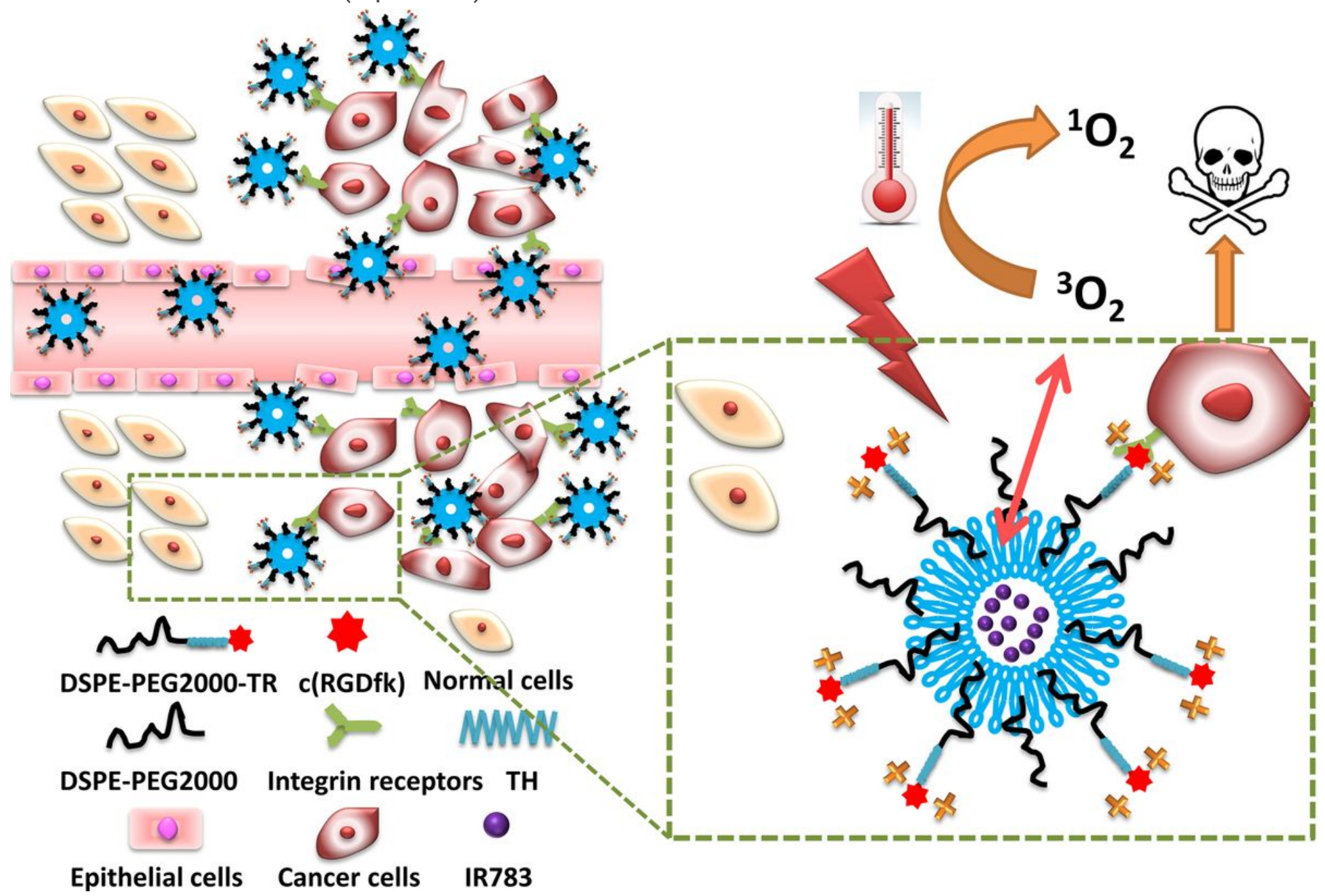

Figure 6

IR783 encapsulated in TR-conjugated liposomes for enhancing NIR imaging-guided PTT and PDT

\section{Supplementary Files}

This is a list of supplementary files associated with this preprint. Click to download.

- Scheme1.tif

- Supplementary.pdf

- GraphicalAbstract.jpg 